\title{
PROGRAMA DE RESILIÊNCIA: PRÁTICAS EDUCATIVAS PARA A PREVENÇÃO DE BULLYING E PROMOÇÃO DA SAÚDE MENTAL NA ADOLESCÊNCIA
}

\section{RESILIENCE PROGRAM: EDUCATIONAL PRACTICES FOR BULLYING PREVENTION AND MENTAL HEALTH PROMOTION IN ADOLESCENCE}

\author{
Tânia Higa Sakuma', Maria Sylvia de Souza Vitalle²
}

\section{RESUMO}

Vários estudos comprovam que intervenções educativas ou programas de resiliência para adolescentes previnem sintomas de depressão e ansiedade, reduzem problemas de condutas, melhoram a aprendizagem e promovem um comportamento para uma vida mais saudável. Existem vários programas de resiliência, de desenvolvimento socioemocional e para prevenção do bullying e da violência entre adolescentes em outros países, mas no Brasil ainda temos muita carência destes tipos de programas. Este artigo apresenta uma pesquisa-ação desenvolvida para elaborar uma proposta de intervenção educativa para fomentar a resiliência em adolescentes de 11 a 18 anos de diferentes contextos e repertórios culturais. Como resultado, foi possível elaborar um programa de resiliência por meio do coaching e de intervenções educativas, aumentando o repertório de práticas para o desenvolvimento de crenças e comportamentos positivos dos adolescentes. Dentro da visão da bioecologia do desenvolvimento humano, estas intervenções incluíram a participação dos adolescentes, das famílias e dos educadores com significativos resultados na construção da resiliência e de comportamentos que previnem a violência e o bullying, como também, promovem a saúde mental e o bem-estar.

PALAVRAS-CHAVE: Resiliência Psicológica. Comportamento do Adolescente. Bullying. Competências Sócioemocionais. Saúde do Adolescente

\section{ABSTRACT}

Several studies have shown that educational interventions or adolescent resilience programs can prevent symptoms of depression and anxiety, reduce behavioral problems, improve learning, and promote behavior toward a healthier life. In several countries, there are several resilience programs for social and emotional development and for prevention of bullying and violence among adolescents, but in Brazil, we still have a great lack of this kind of programs. This article presents an action research developed to elaborate a proposal of educational intervention to foster resilience in adolescents of 11 to 18 years of different contexts and cultural repertoires. As a result, it was possible to develop a resilience program through coaching and educational interventions, increasing the repertoire of practices for the development of positive beliefs and behaviors of adolescents. Within the vision of the bioecology of human development, these interventions included the participation of adolescents, families and educators with significant results in the construction of resilience and behaviors that prevent violence and bullying, as well as promote health and well-being.

KEYWORDS: Psychological Resilience. Adolescent behavior. Bullying. Social and Emotional Competence. Adolescent Health

1 Mestre em Ciências pelo Programa de Pós-Graduação de Educação e Saúde na Infância e Adolescência (ESIA) da Unifesp - Universidade Federal de São Paulo; Doutoranda do ESIA, Unifesp.

2 Prof. Adjunto Dr e Chefe do Setor de Medicina do Adolescente, Unifesp; Professor Permanente do Programa de PósGraduação Educação e Saúde na Infância e Adolescência, Unifesp. Autor correspondente: Endereço: Rua Botucatu, 715, Vila Clementino, CEP 04023-062, São Paulo, SP, Brasil. E-mail: sylviavitalle@gmail.com 


\section{INTRODUÇÃO}

A adolescência é um período de experimentação e autodescoberta marcado por esforços do adolescente de estabelecer sua identidade e autonomia, enfrentando transformações físicas, psicológicas, sociais, cognitivas e culturais. Se estas transformações resultarão em vulnerabilidade (riscos) ou resiliência (proteção), depende da forma como o adolescente pode lidar com as adversidades e criar interpretações positivas ao longo das suas vivências. Podemos considerar a vulnerabilidade como a predisposição do indivíduo em desenvolver problemas de comportamento, problemas de desenvolvimento ou de saúde mental. Por outro lado, a resiliência pode ser considerada como predisposição para superar os eventos negativos e desenvolver-se adequadamente ( PESCE, 2004).

Nesta fase abre-se, assim, um leque de desafios e situações de vários contextos, que tanto podem resultar no amadurecimento e aquisição de habilidades socioemocionais, como também podem gerar questões de desajuste comportamental e emocional causando consequências negativas à saúde. De acordo com Bekaert ( 2003) é na adolescência que se adquire consistência às fundações da saúde futura, aos estilos de vida, às atitudes e aos padrões de comportamento que podem garantir ou não o bem-estar e uma melhor realização da pessoa.

Em 2012, a OMS - Organização Mundial de Saúde publicou um estudo que constata que $20 \%$ dos adolescentes no mundo enfrentam problemas de saúde mental, mas permanecem diagnosticados e tratados de forma inadequada. A causa principal destes transtornos estão relacionadas às suas condições de vida, estigma, discriminação ou exclusão, violência ou bullying, além de falta de acesso a serviços e apoio de qualidade (OMS, 2012). Estes dados atualizados em 2018, indicam que a depressão e ansiedade são as principais causas de doenças e incapacidades entre os adolescentes a partir dos 14 anos e, que o suicídio é uma das principais causas da morte nesta mesma faixa etária (OMS, 2018).

De acordo com estes dados alarmantes, podemos afirmar que adolescência traz implícita a necessidade de programas preventivos ou intervenções educativas que visam o desenvolvimento socioemocional, da resiliência e das competências de vida (DIAS, CRUZ e DANISH, 2001). A intervenção educativa está relacionada aos conceitos de educação em saúde, que pode ser compreendida como "combinações de experiências de aprendizagem planejadas para facilitar ações voluntárias conducentes à saúde". Este conceito de Candeias ( 1997), enfatiza a importância de combinar diversos aspectos determinantes do comportamento humano com diversas experiências de aprendizagem, por meio de uma intervenção educativa sistematicamente planejada, para facilitar ou possibilitar ações de um indivíduo, grupo ou comunidade, para alcançar um efeito positivo e intencional sobre a própria condição de bem-estar e de saúde física, mental e emocional.

$\mathrm{Na}$ maioria dos programas de resiliência, os adolescentes são estimulados a adquirir competências, gerir emoções, criar autonomia, estabelecer e desenvolver relações interpessoais mais maduras e desenvolver integridade pessoal (DANISH, FORNERIS e WALLACE, 2005). Pesquisadores da Universidade da Pensilvânia (SELIGMAN, 2007) desenvolveram vários estudos de promoção da resiliência em crianças e adolescentes, que comprovam que Programas de Resiliência para adolescentes previnem sintomas de depressão e ansiedade, reduz problemas de condutas, melhora a aprendizagem, promove um comportamento para uma vida mais saudável e favorece o bem-estar dos adolescentes ( SELIGMAN, 2007).

O programa desenvolvido na Universidade da Pensilvânia chama-se PRP (Penn Resilience Program) e tem como objetivo a prevenção da depressão de adolescentes (GILLHAM, 2006). Seligman (2007), mentor do programa, sugere que o incremento de habilidades comportamentais resilientes seja obtido pelo reforço positivo dos comportamentos desejáveis, favorecendo o sentimento de competência, de identificação de aspectos positivos nas experiências de vida, no fortalecimento da autoestima e de modelos de estilos explicativos positivos oferecidos por adultos significativos. Muitos outros programas reconhecidos nos Estados Unidos reforçam as competências emocionais e habilidades sociais das crianças e adolescentes (GILLHAM, 2006). Em 2017, os resultados de uma pesquisa americana realizada com adolescentes de 12 a 17 anos revelaram que a resiliência é um potente fator de proteção, tanto na prevenção do bullying ou 
cyberbullying como também na atenuação dos seus impactos prejudiciais nas vítimas (HINDUJA e PATCHIN, 2017).

Nesta última década, muitos outros programas educativos de resiliência, presenciais na escola ou por mídias digitais, foram desenvolvidos em várias partes do mundo para desenvolver competências sociais e emocionais, como também, para reduzir a violência e o bullying.

O programa anti-bullying KiVa (2018) foi desenvolvido pela Universidade de Turku na Finlândia e financiado pelo Ministério da Educação e Cultura. O programa foi implementado em mais de $90 \%$ das escolas finlandesas, com estudos relatando redução média de $60 \%$ de atos de bullying ou de vitimização no primeiro ano de adesão. Além disso, foi constatado a redução do número de casos de ansiedade e depressão, favorecendo efeitos positivos no desempenho, motivação, convívio harmonioso e bem-estar dos alunos (SUOMI, 2011). O KiVa vem ganhando visibilidade internacional, sendo aplicado nos países da Europa, como também, em outros continentes. Durante o programa, os alunos são estimulados a refletir sobre valores morais como a empatia e o respeito pelos outros por meio de dinâmicas, jogos, filmes, videogames e muito diálogo sobre o assunto. A equipe de educadores da escola é orientada para identificar casos de agressão verbal, física ou por mídias eletrônicas e desenvolver diálogos individuais ou em grupo. São também utilizadas caixas de correio para denunciar a prática de bullying na escola ( TURKU, 2018)

A Austrália é um dos países que mais desenvolveu programas de resiliência e bem-estar em ambientes escolares. Em 2017, o governo australiano também criou o Programa Nacional de Apoio à Saúde Mental da Criança e do Jovem que integrou várias iniciativas independentes e consolidou o "Be You" que é um programa de suporte de aprendizagem online para educadores e outros profissionais aprimorarem as suas próprias competências para desenvolver a resiliência e a saúde mental das crianças e adolescentes( BLUE, 2019).

No ano de 2017, o Japão registrou aproximadamente 415.000 casos de bullying nas escolas (DIPLOMAT, 2019) e em 2018 o Ministério da Educação anunciou o maior índice de suicídio entre adolescen- tes japoneses nos últimos 30 anos ( CNN, 2018), ou seja, em apenas um ano, 250 estudantes perderam as suas vidas em decorrência ao bullying, cyberbullying, estresse emocional e questões familiares. Paralelamente, não foram identificados programas de resiliência psicológica para crianças e adolescentes no Japão. De acordo com pesquisadores da Universidade de Fukuoka, somente em 2013 que começaram os primeiros estudos sobre programas de aprendizagem socioemocional para os estudantes (KOIZUMI, 2016).

Os programas de resiliência ajudam o adolescente a obter recursos para desafiar as situações de seu cotidiano e promover seu bem-estar. No entanto, como qualquer outra ferramenta, a sua adequação e o seu valor estão na capacidade de saber utilizá-la. A ideia não é cultivar a sobrevivência dos adolescentes melhores, mais fortes e competitivos, mas sim, oferecer novas possibilidades para os adolescentes lidarem com situações mais adversas da vida, manifestando o melhor do seu potencial. É importante observar os contextos culturais e específicos de cada indivíduo, para não inculcar conceitos e valores hegemônicos típicos de sociedades majoritárias (UNGAR, 2008). Dentro de uma visão mais humanista do desenvolvimento do adolescente, faz-se necessário construir a resiliência considerando a ética, a igualdade, equilíbrio ecológico e ambientes humanos sustentáveis. Além destes programas de resiliência e de desenvolvimento da aprendizagem socioemocional para crianças e adolescentes foram desenvolvidos também programas que ajudem os pais e as escolas a promover a resiliência dos jovens (WONG, 2009).

A elaboração do Programa de Resiliência deste estudo foi baseada na Bioecologia do Desenvolvimento Humano de Bronfenbrenner (2011). O contexto primordial de desenvolvimento, segundo o autor, é aquele em que o indivíduo pode engajar-se em atividades conjuntas cada vez mais complexas, com a orientação direta de pessoas, que se comprometam com seu bem-estar e com quem ele tenha uma relação afetiva positiva. Essas interações acentuam, encorajam e oferecem as condições para que ele possa explorar outros recursos sociais e físicos imediatos e o ambiente simbólico que convida à exploração e à elaboração, envolvendo novas experiências. Progressivamente, essas interações se defrontam com os valores e as crenças da socieda- 
de em que vive, por meio de referências do ambiente doméstico, dos programas educativos, do trabalho de seus pais, etc. Enfim, o efetivo processo de desenvolvimento do adolescente, segundo esta teoria, requer que existam políticas e programas que possam dar-lhe estabilidade, condições e reconhecimento de todas as instâncias anteriores, incluindo-se as políticas econômicas e os valores sociais das instituições pelas quais ela circulará em direção a seu futuro.

Diante do exposto, justifica-se a realização desta pesquisa-ação com o objetivo de elaborar dados e experiências para o desenvolvimento de um Programa de Resiliência, com base na Bioecologia do Desenvolvimento Humano, como uma prática de desenvolvimento social e emocional para promover interações harmoniosas, saúde e bem-estar nos adolescentes brasileiros. Este Programa de Resiliência é direcionado para os próprios adolescentes, como também para os pais, educadores e pessoas da comunidade ao seu entorno, visando desencadear mudanças do comportamento individual ou do grupo, para promover a saúde e a cultura de paz em escolas, abrigos, associações, clubes, entre outros ambientes ou contextos.

\section{Percurso Metodológico}

Este estudo faz parte de um projeto maior intitulado "Construção da Resiliência do Adolescente: Uma Abordagem Bioecológica" aprovado pelo Comitê de Ética em Pesquisa da Universidade Federal de São Paulo, Número do Parecer: 354.900 de 09/08/2013. O desenvolvimento foi elaborado na forma de pesquisa -ação para compreender o fenômeno da resiliência na adolescência e elaborar uma proposta de intervenção educativa em diversos tipos de contextos. A pesquisa-ação é um tipo de pesquisa participante engajada que procura unir a pesquisa à ação ou prática, isto é, desenvolver o conhecimento e a compreensão como parte da prática. A pesquisa-ação pode ser aplicada em qualquer ambiente de interação social que se caracterize por um problema, no qual estão envolvidos pessoas, tarefas e procedimentos.

Os passos desta pesquisa foram: 1) Mapeamento da condição de resiliência dos adolescentes; 2) Revisão de Literatura sobre programas de resiliência, de desenvolvimento socioemocional e de bullying para elaboração da intervenção educativa; 3) Aplicação da intervenção educativa para adolescentes, pais, educadores; 4) Consolidação das teorias e experiências adquiridas durante a pesquisa-ação; 5) Proposta do programa de resiliência que estimula as habilidades socioemocionais e reduz conflitos adolescentes.

Instrumento: Esta pesquisa utilizou, como instrumento para o Mapeamento da Resiliência, a escala de resiliência "Quest_Resiliência", que possibilita mapear a intensidade das crenças que organizam o comportamento resiliente e através destes dados, podemos identificar padrões de pensamentos e atitudes nas situações de maior estresse. A intensidade podese configurar como um comportamento de passividade diante das situações adversas, com uma predominância em "acatar" e "absorver" o impacto que o estresse provoca; como também, pode-se configurar como um comportamento de intolerância evidenciando crenças com uma predominância em "rejeitar" ao impacto do estresse (BARBOSA, 2011)

Esta escala foi validada no Brasil como "Questionário do Índice de Resilência: Adulto Reivich-Shatté/ Barbosa" por Barbosa (2006). Após muitas investigações e pesquisas científicas, este instrumento foi ampliado em 2009, passando a se chamar "Quest_Resiliência". Como embasamento científico, esta escala conta com contribuições da abordagem Psicossomática, da Psicologia Positiva e a da Teoria Geral dos Sistemas. No entanto, está estruturada nos pressupostos teóricos da Terapia Cognitiva Comportamental, (TCC), cobrindo oito Modelos de Crenças Determinantes (MCDs) relacionados com a resiliência: autoconfiança, autocontrole, leitura corporal, análise de contexto, conquistar e manter pessoas, empatia, otimismo e sentido de vida. A escala "Quest_Resiliência" possibilita o mapeamento dos modelos de crenças e permite constatar a existência de crenças conflitantes dos respondentes permitindo uma visualização de possíveis ressignificações para potencializar as áreas de "fragilidade" e obter uma condição de "excelência em resiliência".

O uso do Quest_Resiliência requer autorização formal da Sociedade Brasileira de Resiliência (SOBRARE) que, atualmente, detém os direitos de cessão de todas as versões utilizadas como ferramentas para pesquisas acadêmicas. Portanto, para esta pesquisa foi formalizado um contrato de cessão de uso 
para aplicação e tabulação de dados pelo sistema on -line do Quest_Resiliência versão para Adolescentes (QRA), através de contrato entre as pesquisadoras com a SOBRARE.

Sujeitos: Neste estudo, escolhemos duas amostras de adolescentes em contextos diferentes (ambiente de vulnerabilidade e de proteção), ambos os sexos, de idade aproximada de 11 a 18 anos selecionados por conveniência, contando também com a participação de educadores, pais ou responsáveis que atuam no entorno destes adolescentes. As características dos contextos de cada amostra são:

Amostra 1: Adolescentes que frequentam uma escola particular de cursos livres da Zona Sul da cidade de São Paulo, que tem uma forte influência da cultura japonesa, pois dentro das suas várias opções de cursos oferecem: Curso de Língua e Cultura Japonesa, Curso de Soroban (ábaco japonês) e Curso de Desenho Mangá. Tivemos a participação de 48 adolescentes, sendo que do sexo feminino participaram 15 indivíduos (10 meninas entre 11 a 14 anos e 5 meninas acima de 15 anos) e do sexo masculino participaram 33 indivíduos (20 meninos de 11 a 14 anos e 13 meninos acima de 15 anos). Do total de 48 indivíduos, 68,75\% são descendentes de japoneses. Os outros $31,25 \%$, mesmo não sendo descendentes de japoneses, simpatizam-se e interessam-se pela cultura oriental. Estes adolescentes moram com os pais biológicos, são estudantes de escolas particulares e têm acesso à formação complementar (cursos livres de línguas estrangeiras, informática, esportes e cursos para desenvolvimento cognitivo). Todos possuem convênio médico particular e não têm indícios de uso de drogas.

Amostra 2: Adolescentes abrigados pelo Serviço de Acolhimento Institucional para Crianças e Adolescentes vinculado à Secretaria de Desenvolvimento e Assistência Social da Prefeitura do Município de Guarulhos - SP. Estes adolescentes foram acolhidos por abandono, maus-tratos, situação de rua, violência sexual intrafamiliar, genitores em privação de liberdade, genitores usuários de drogas, entre outros motivos. Tem acesso à Saude através do SUS - Sistema Único de Saúde e estão matriculados nas escolas públicas próximas às casas de abrigo. A todos os acolhi- dos maiores de quatorze anos são oferecidos cursos profissionalizantes e oportunidades para a inclusão no mercado de trabalho. No entanto, a baixa escolaridade e pouca adesão, principalmente por parte dos acolhidos usuários de drogas, prejudicam a inclusão e a continuidade dos mesmos nas atividades. Participaram deste estudo 22 adolescentes, originários de cinco casas de abrigo de regiões diferentes do Município de Guarulhos, sendo 5 indivíduos do sexo feminino (2 meninas entre 11 a 14 anos e 3 meninas acima de 15 anos) e 17 indivíduos do sexo masculino (8 meninos de 11 a 14 anos e 9 meninos acima de 15 anos).

\section{Desenvolvimento}

Após a autorização dos pais e dos adolescentes para a participação neste estudo, os adolescentes da Amostra 1 foram divididos em pequenos grupos de acordo com a disponibilidade de horário para participarem dos encontros. Em cada grupo foram aplicados o Quest_Resiliência Adolescentes (QRA) e os resultados da tabulação foram organizados, via on-line pelo sistema da SOBRARE, em oito grandes áreas de Modelos de Crenças Determinantes (MCD): Autocontrole, Autoconfiança, Análise de Contexto, Leitura Corporal, Conquistar e Manter Pessoas, Empatia, Otimismo com a Vida e Sentido de Vida. De acordo com estes resultados foi possível avaliar os pontos fortes e fracos em cada MCD (categorizados em excelente, forte, bom, moderado, fraco), como também, foi possível analisar o estilo predominante de comportamento resiliente (passivo ou intolerante ao processo de estresse) de cada indivíduo e de cada grupo de adolescentes no conjunto.

O conteúdo para o programa preliminar foi elaborado a partir da revisão de literatura, como também, de acordo com as necessidades e possibilidades de cada grupo de aplicação. O mesmo conteúdo programático foi aplicado para todos os grupos. A ordem dos temas também pôde ser alterada para atender à necessidade e o interesse dos grupos.

Os encontros educativos com os adolescentes da Amostra 1 foram organizados em 7 turmas (4 grupos de 11 a 14 anos e 3 grupos de adolescentes acima de 15 anos). Cada grupo tinha em média 7 integrantes. Os encontros educativos com os pais, familiares e educadores da Amostra 1 foram organizados em 8 
turmas de pais e 2 turmas de educadores. Cada turma teve em média 8 participantes, com a participação total de 59 pais e 12 educadores.

A operacionalização da intervenção educativa dos adolescentes da Amostra 2 teve um formato diferente, pois pela dificuldade de organização de pequenos grupos de adolescentes, por diversos motivos como: logística de horários (cada um estuda ou trabaIha em horários diferentes), logística física (distância entre as casas) e, o mais importante, pelo levantamento dos dados obtidos, os adolescentes do abrigo tiveram índices altos de problemas comportamentais e de saúde mental. Para não os expor foi decidido que a intervenção deles seria realizada num processo de coaching individual com os psicólogos e/ou coordenadores das casas de abrigo. Desta forma, foram coletados os dados para o Quest_Resiliênia pelos psicólogos e o Programa de Resiliência foi iniciado primeiramente, com a equipe profissional das casas de abrigo (educadores, cuidadores, psicólogos, assistentes sociais, motoristas, cozinheiras e seguranças). Nas intervenções educativas com a equipe profissional, a participação total foi de 76 pessoas. A divisão das turmas foi organizada pelos coordenadores das casas de abrigo, considerando a disponibilidade de cada participante para uma ação educativa de 6 horas. Todos os participantes estavam dispostos a encontrar novas possibilidades de lidar com os adolescentes, de uma forma mais leve e com mais comunicação.

\section{RESULTADOS}

\section{Programa de Resiliência - Adolescentes}

Durante esta pesquisa-ação, as pesquisadoras elaboraram, aplicaram e trouxeram melhorias nos temas e nas dinâmicas de aplicação, pela interação com os adolescentes, seus pais, familiares e seus educadores. As atividades, os jogos e dinâmicas organizados para a aplicação do conteúdo tinham como base:

a) Estímulo e desenvolvimento de processos ou habilidades cognitivas básicas essenciais para o pleno desenvolvimento intelectual/ cognitivo, a fim de potencializar a aprendizagem, o desempenho escolar e habilidades profissionais. b) Estímulo e desenvolvimento de processos ou habilidades socioemocionais que engloba autoconhecimento, controle dos sentimentos, de automotivação, de empatia, de habilidade sociais e de direcionamento de vida.

\section{Objetivos e Temas para adolescentes}

Os objetivos da intervenção educativa específicos para os adolescentes foram:

- Conscientizar os adolescentes sobre a resiliência, os benefícios da construção da resiliência e como pode ser a atuação deles mesmos para potencializar este processo.

- Promover técnicas de construção da resiliência através do autoconhecimento (autocontrole, autoconfiança, leitura corporal, autorregulação), habilidades sociais (empatia, comunicação, relacionamento interpessoal), atitudes positivas (análise de contexto, otimismo, sentido de vida), entre outros temas.

\section{Temas Propostos - Adolescentes}

Tema 1 - Resiliência e Flexibilidade: A capacidade das pessoas em acreditar na sua própria eficácia e tomar iniciativas contribui significativamente para a sua capacidade de flexibilidade e de se recompor diante das adversidades da vida. Este encontro proporciona uma concreta demonstração do conceito de flexibilidade e as formas de como a flexibilidade mental influencia nos relacionamentos.

Tema 2 - Empatia e Relacionamentos: Empatia é a habilidade de compreender a perspectiva e o estado emocional dos outros em seu estado emocional e psicológico, como também, agir de acordo com esse entendimento. Demonstrar empatia é a base da construção de relacionamentos significativos. Este encontro foca nas formas de estabelecer e melhorar a capacidade de empatia e a habilidade de responder apropriadamente à situação de outra pessoa.

Tema 3 - Pensamento Otimista: Uma perspectiva positiva conduz a uma felicidade maior e mais interações sociais mais bem-sucedidas. Este encontro ensina a diferença entre pensamentos pessimistas e otimistas e mostra aos participantes meios de substi- 
tuir pensamentos pessimistas por pensamentos mais otimistas, mas realistas ao mesmo tempo.

Tema 4 - Ansiedade, Raiva e Medo (Leitura Corporal): Uma perspectiva sobre o que é a ansiedade, a raiva e o medo e como estes sentimentos influenciam no nosso corpo, pensamentos e atitudes. A Leitura Corporal é a capacidade de perceber as mudanças que acontecem em seu corpo diante de situações adversas para controlar os "sintomas do estresse" nos sistemas nervoso e muscular.

Tema 5 - Autocontrole e Autorregulação: Autocontrole das emoções se refere à capacidade da pessoa se manter calma e administrar seus sentimentos quando está sob pressão ou diante de imprevistos. O termo autorregulação refere-se aos inúmeros processos de pensamento que gerenciam os nossos impulsos e emoções. A autorregulação ajuda-nos a aceder aos raciocínios que nos permitem ser assertivos, inibindo os impulsos primários e orientando a nossa atenção para a construção de uma resposta adequada à situação.

Tema 6 - Autoestima e Autoconfiança: A autoestima é a opinião e o sentimento que cada pessoa tem por si mesma, é a capacidade de respeitar, acreditar e amar a si mesma. A autoestima também é a apreciação que uma pessoa faz de si mesma em relação à sua autoconfiança e seu autorrespeito. Através dela podemos enfrentar desafios e defender nossos interesses. A autoconfiança é a habilidade de sentir eficaz nas ações que serão realizadas. Acreditar em seus recursos internos.

Tema 7 - Percepcõos e Análise de Contexto: Perceber as situações e analisar o contexto das adversidades é uma competência muito importante no desenvolvimento da resiliência. Análise de contexto se refere à habilidade de identificar as causas dos problemas e adversidades e tomar atitudes proativas.

Tema 8 - Escolhas e Sentido de Vida: Saber que podemos ter escolhas nas situações de desafios, nos faz sentir mais fortes. Assim podemos enfrentar as dificuldades de uma forma mais efetiva. Criar alternativas para resolução de problemas influencia positivamente na forma como reagimos. Esta sessão foca em ajudar os participantes a fazerem escolhas proativas. Gerar escolhas requer flexibilidade no pensamento. Sentido de vida é a capacidade de entender e manter um senti- do maior para a existência, trazendo valor para a vida. As escolhas estão alinhadas aos valores e o sentido de vida de cada um.

\section{Programa de Resiliência - Pais/Familiares e Educadores}

Os objetivos da proposta de intervenção educativa específicos para os pais, familiares e educadores foram conscientizá-los sobre a resiliência, os benefícios da construção da resiliência nos adolescentes e como pode ser a atuação deles para potencializar este processo. O conteúdo programático foi desenhado para 3 horas para os familiares e 6 horas para os educadores, mas por ser um processo de conscientização, esta carga horária pode ser subdividida ou readequada de acordo com a disponibilidade de participação das pessoas envolvidas.

\section{Conteúdo Proposto - Pais, familiares e educadores}

Como conteúdos específicos para os pais e educadores foram propostos os seguintes pontos:

1. Conceito de Resiliência

a. Conhecer o conceito de resiliência, a sua origem da Física e como o conceito é aplicado na área comportamental;

b. Identificar os benefícios da resiliência em si mesmo;

c. Perceber o impacto e a importância da resiliência para os adolescentes.

2. Características comportamentais dos adolescentes na atualidade

a. Discutir sobre como pensam as diferentes gerações;

b. Discutir sobre o padrão de comportamento dos adolescentes em geral e especificamente na instituição.

3. A atuação do educador no desenvolvimento da resiliência do adolescente

a. Reconhecer os diversos tipos de papéis e funções do educador;

b. Aprimorar as formas de comunicação com os adolescentes;

c. Conhecer o conceito do processo de Co- 
aching;

d. Aprender algumas técnicas do processo de Coaching;

e. Compreender como funciona os modelos de crenças.

4. Os pontos que podem ser trabalhados com os adolescentes

a. Resiliência e Flexibilidade

b. Empatia e Relacionamento

c. Pensamento Positivo

d. Ansiedade, Raiva e Medo (Leitura Corporal)

e. Autocontrole e Autorregulação

f. Forças Pessoais, Autoestima e Autoconfiança

g. Escolhas e Sentido de Vida

5. Meios para estimular a resiliência dos adolescentes
a. Interações e Diálogos
b. Jogos e Dinâmicas
c. Metáforas e Histórias
d. Atividades e Desafios do Cotidiano
e. Referências Positivas

\section{Resultados da Aplicação do Programa}

No final do programa de cada grupo da Amostra 1 , foi apresentado aos adolescentes, os pontos vivenciados em cada encontro articulando-os às habilidades aprendidas documentadas por portfolio de fotos ou registros de observação. Os adolescentes se sentiram muito orgulhosos de suas conquistas, perceberam o quanto a sua autoestima e resiliência foram fortalecidas e solidificadas no decorrer da intervenção. Como avaliação da intervenção educativa, foi pedido comentários sobre o que eles aprenderam, o que eles perceberam de mudanças e qual o impacto destas mudanças no seu meio escolar, familiar e social. A maior parte dos adolescentes disseram que mudaram a sua forma de pensar e perceber a realidade deles, eles também disseram que estavam mais calmos e mais seguros para uma melhor interação com os pares e para os desafios do dia-a-dia. Durante os primeiros encontros, o propósito era formar vínculos, integração e motivação. Os passos seguintes foram identificar como se apresentavam os comportamentos e as crenças mapeadas na fase anterior e, avaliar a prontidão do adolescente para o desenvolvimento da intervenção educativa. Muitas vezes, houve a necessidade de incorporar outros tipos de atividades de acordo com a característica e o interesse de cada grupo. Um ponto importante foi facilitar o processo de descoberta orientada, através do processo de Coaching, onde o participante foi ajudado a reavaliar seus pensamentos, crenças e suposições e, a desenvolver cognições e processos cognitivos alternativos, mais equilibrados, funcionais e úteis. Este processo de autodescoberta e de promoção da autoeficácia foi facilitado através de diálogos, nos quais os adolescentes foram ajudados a perceber informações novas ou ignoradas anteriormente. Usou-se também uma variedade de perguntas, cada uma com um foco diferente, que ajudaram o adolescente a identificar e testar sistematicamente os seus pensamentos.

Neste modelo de construção de resiliência, o propósito foi de abrir caminhos para que os adolescentes pudessem perceber as suas próprias potencialidades, fortalecendo competências e habilidades úteis para a aprendizagem e construindo crenças ou esquemas de pensamentos saudáveis e enriquecedor para sua saúde mental e bem-estar. Os encontros foram planejados com apresentação das atividades de forma lúdica, numa sequência prática e dinâmica, com equilíbrio entre diálogos, realização de jogos, dinâmicas de grupo e exercícios graduados para o desenvolvimento das técnicas e habilidades, de acordo com a faixa etária de cada grupo.

A estratégia de aplicação do Programa de Resiliência da Amostra 2 foi com o foco de intervenção do entorno dos adolescentes. A expectativa foi intervir junto aos educadores da casa de abrigo para criar novas referências positivas e facilitar a posterior intervenção junto aos adolescentes. Os educadores e a equipe de profissionais ficaram bastante entusiasmados com os novos aprendizados sobre resiliência, técnicas de comunicação mais efetiva com os adolescentes, como também, formas de aliviar o próprio estresse. Percebeu-se bastante abertura para novas possibilidades de mudanças no ambiente dos abrigos. Mesmo não atuando diretamente com os adolescentes, ao longo do processo, pode-se observar que a interação de um adulto consciente da sua própria possibilidade de promover resiliência dos adolescentes tem aberto diálogos e am- 
bientes mais saudáveis para os adolescentes dos abrigos. O próximo passo será a realização da capacitação dos psicólogos e coordenadores das casas de abrigo para atuar com os conteúdos propostos através do processo de coaching individual junto aos adolescentes, potencializando desta forma a sua resiliência.

\section{CONCLUSÃO}

A ação educativa com adolescentes de diferentes contextos não tem a finalidade de torná-lo iguais, mas sim, de dar a todos a oportunidade de aprender, crescer e promover a sua resiliência para uma vida plena e feliz, de acordo com a realidade e possibilidade de local de atuação. Consideramos a resiliência como uma aprendizagem em construção permanente, ou seja, um processo dinâmico de desenvolvimento humano que se fortalece a partir das relações do indivíduo com o seu meio, ao longo da vida.

O modelo bioecológico do desenvolvimento humano de Bronfenbrenner (2011) oferece uma visão das possibilidades de expansão desse desenvolvimento e da perspectiva educativa dos diversos ambientes na educação dos sujeitos. Sua percepção sobre a necessidade de participação e de interação das pessoas (crianças e adolescentes) com outras e com novos ambientes considera que essas diferentes interações são desafios que provocam seu crescimento. Analisa, ainda, aspectos não visíveis que determinam o desenvolvimento humano, como as crenças, os valores e os elementos da cultura local e universal, vistos de forma integrada no cenário da vida dessas pessoas. De acordo com a pessoa, processo, tempo e contexto cada experiência pessoal traz novos significados na reflexão e na tomada de consciência. Assim, um processo de potencialização da resiliência deve ser um projeto para a vida: onde o pensar - sentir - agir também esteja conectado com outras realidades.

O envolvimento dos pais e de educadores no processo de promoção de resiliência de adolescentes funcionaram como fatores de reforço, pois a influência dos adultos no desenvolvimento de comportamentos e cognições funcionais e disfuncionais do adolescente foi reconhecida e incluída na formulação dos encontros.

a. Os pais e os educadores puderam aprender como poderiam ajudar e apoiar a promoção da resiliência dos adolescentes. Eles entenderam que podem transmitir aos adolescentes mensagens consistentes sobre a importância e o valor das novas habilidades de comportamento que estão aprendendo.

b. Eles também entenderam que a prática das novas habilidades fora da intervenção educativa pode ser monitorada, estimulada e reforçada pelos pais e/ou educadores.

c. Importantes percepções, expectativas e crenças dos pais e dos educadores sobre o adolescente puderam ser contestadas e reavaliadas.

d. Foram redirecionados os comportamentos ou crenças dos pais e educadores que poderiam limitar o adolescente a aprender novas habilidades.

e. A continuidade de pontos de melhoria e a manutenção das habilidades aprendidas após a intervenção educativa podem ser facilitadas e reforçadas pelo envolvimento dos adultos que estão como referência no entorno do adolescente.

No processo de desenvolvimento da pesquisa -ação foi possível verificar na prática algumas novas tendências dos estudos da resiliência, enumerados a seguir:

1) Muitos pesquisadores pensavam que os adolescentes de nivel socioeconômico alto fossem mais resilientes. No entanto, o que podemos perceber é que esses adolescentes podem ser mais bem-sucedidos na escola. No entanto, devemos entender que bom desempenho escolar não significa resiliência. O nível socioeconômico baixo não impede o desenvolvimento da resiliência, do crescimento e desenvolvimento humano. $\mathrm{O}$ estudo com as duas amostras analisadas, de classes socioeconômicas bem diferentes, comprovou esta característica da resiliência. Podemos afirmar também que a resiliência é diferente dos fatores de risco e proteção.

2) Contextualizar a promoção da resiliência de acordo com a fase de desenvolvimento da vida do índivíduo, permite ter um direcionamento para promover novas habilidades e competências sobre base já desenvolvida em etapas anteriores. Então, podemos 
entender que, a resiliência está ligada ao desenvolvimento e ao crescimento humano, incluindo diferenças etárias e de gênero. Nesta pesquisa, também foi evidenciado que elaborar uma intervenção educativa focada na realidade e necessidade dos adolescentes traz produtividade na promoção e desenvolvimento de novas competências.

3) Programas de Resiliência engessados com conteúdos e aplicações fixas podem não atingir o interesse e a conscientização dos adolescentes e das pessoas do seu entorno. Por meio das interações e ações educativas criativas junto aos adolescentes e na interação com os pais e educadores, foi constatado que promover a resiliência e comportamentos resilientes requerem diferentes estratégias como jogos e brincadeiras, dinâmicas, contação de histórias, diálogos, discussões dirigidas e o processo de coaching.

4) Com a utilização de instrumentos como o Quest Resiliência, foi possível comprovar que a resiliência pode ser medida e promovida e, além disso, está relacionada com saúde mental e qualidade de vida. Por meio das intervenções em grupo e individuais, podemos perceber que a resiliência é um processo em que é possível trabalharmos com a sua promoção do bem-estar e qualidade de vida como um processo educativo e como práticas de políticas públicas.

Ao longo da pesquisa-ação, pudemos compreender que a resiliência só existe na medida em que a bioecologia física e social de um adolescente estão ao seu alcance, ou seja, os indivíduos, a família, a comunidade e os recursos sociais precisam estar combinados e presentes, para que numa ação conjunta promova a resiliência a favor de resultados positivos e saudáveis.

As pesquisas no Brasil sobre o processo de promoção de resiliência nos adolescentes relacionadas com o desenvolvimento das competências socioemo- cionais e com o propósito de reduzir as experiências de bullying ainda são bem escassas. Ao considerar a resiliência como um processo passível de ser promovido nos adolescentes, teorias e conceitos mais convergentes tornam-se cada vez mais necessários. Assim, os pesquisadores e profissionais de diversas áreas podem se unir e elucidar as dinâmicas subjacentes e intervenções mais eficazes deste processo de construção. Outro fator a ser considerado é o foco das teorias e pesquisas no conceito de resiliência do adolescente, para que se possa sistematizar intervenções de promoção psicossocial e adaptação positiva com base na cultura e nos múltiplos cenários em que o adolescente está inserido.

As futuras pesquisas e os programas de promoção de resiliência de adolescentes podem ser planejados seguindo conceitos mais claros de resiliência e a conexão entre os diferentes componentes do seu processo, contemplando ainda, as variações culturais que afetam o processo de interação do indivíduo e o seu contexto. O desafio contínuo de visualizar diretrizes comuns na promoção do desenvolvimento humano, da convivência harmoniosa e da saúde pública, permite aumentar a comunicação entre as diferentes disciplinas, aumentando, consequentemente, a possibilidade de mais pesquisas interdisciplinares com aportes de antropólogos, sociólogos, educadores, biólogos, psicólogos, médicos, especialistas de diversas áreas e dos próprios jovens para, assim, poder explicar e desenvolver o processo de resiliência dos adolescentes de forma eficaz e sustentável. $O$ enfoque em resiliência do adolescente permite a promoção da qualidade de vida e bem-estar destes como um trabalho coletivo, multidisciplinar, ético e humano. Dessa forma, a resiliência permite uma nova epistemologia do desenvolvimento humano, pois enfatiza seu potencial, valoriza cada cultura e faz um chamado à responsabilidade coletiva. 


\section{REFERÊNCIAS}

BARBOSA, G. S. Resiliência em Professores do Ensino Fundamental de $5 a$ a 8 a série. Validação e aplicação do "Questionário do Índice de Resiliência: Adultos - Reivich-Shatté/Barbosa" 2006. $330 \mathrm{f}$. Tese (Doutorado em Psicologia Clínica). São Paulo: PUC/SP, 2006.

BARBOSA, G. S. Roteiro dos índices de resiliência Análise comentada do "Quest_Resiliência" versão ambiente de trabalho. São Paulo: Clube dos Autores, 2011.

BEKAERT, S. Developing adolescent services in general practice. Nursisng Standard, v. 17, n. 36, p. 33-36, 2003.

BLUE, B. Learning Resilience. Be You, 2019. Disponivel em: https://beyou.edu.au/learn/learning-resilience. Acesso em: 10 mar. 2019.

BRONFENBRENNER, U. Bioecologia do desenvolvimento humano: tornando os seres humanos mais humanos. Porto Alegre: Artmed, 2011.

CANDEIAS, N. M. F. Conceitos de educação e de promoção em saúde: mudanças individuais e mudanças organizacionais. Revista de Saúde Pública, v. 31, n. 2, p. 209-213, 1997.

CASEL. Guide 2013. Effective Social and Emotional Learning Programs. Preeschool and Elementary School Edition. Chicago: CASEL , 2013.

CNN. Health: Japan's youth suicide rate highest in 30 years. CNN, 2018. Disponivel em: https://edition. cnn.com/2018/11/05/health/japan-youth-suicide-int//index.html. Acesso em: 10 mar. 2019.

DANISH, S. J.; FORNERIS, T.; WALLACE, I. Sport-Based Life Skills Programming in the Schools. Journal of Applied School Psychology, v. 21, n. 2, p. 41-62, 2005.

DIAS, C.; CRUZ, J. F.; DANISH, S. O desporto como contexto para a aprendizagem e ensino de competências de vida: programas de intervenção para crianças e adolescentes. Análise Psicológica, v. 19, n. 1, p. 157-170, 2001.

DIPLOMAT, T. Tokyo Report. The Diplomat, 2019. Disponivel em: https://thediplomat.com/2019/02/ japans-school-bullying-escalates-with-spike-in-youthsuicide/. Acesso em: 17 mar. 2019.
DIX, K. L. . et al. Implementation quality of whole-school mental health promotion and students' academic performance. Child and adolescent mental health, v. 17, n. 1, p. 45-51, 2012.

GILLHAM, J. E. . School-based prevention of depression and anxiety symptoms in early adolescence: A pilot of a parent intervention component. School Psychology Quarterly, v. 21, n. 3, p. 323, 2006.

HINDUJA, S.; PATCHIN, J. W. Cultivating youth resilience to prevent bullying and cyberbullying victimization. Child Abuse \& Neglect, v. 73, p. 51-62, 2017.

KOIZUMI, R. Implementation and Sustaining os Social and Emotional Learning (SEL) Programs in Japan: Application of Anchor Point Planting Approach. The Annual Report of Educational Psychology in Japan, Fukuoka, v. 55, p. 203-217, 2016.

OMS. Adolescent mental health: mapping actions of nongovernmental organizations and other international development organizations. WHO World Health Organization. Switzerland. 2012.

OMS. Folha Informativa - Saúde Mental dos Adolescentes. OPAS Organização Pan Americana de Saúde - OMS Organização Mundial de Saúde, 2018. Disponivel em: https://www.paho.org/bra/index. php?option=com_content $\&$ view $=$ article $\& i d=5779$ :foIha-informativa-saude-mental-dos-adolescentes\&ltemid=839. Acesso em: 10 mar. 2019.

PESCE, R. P. Risco e proteção: em busca de um equilíbrio promotor de resiliência. Psicologia: teoria e pesquisa, v. 20, n. 2, p. 135-143, 2004.

SELIGMAN, M. Florescer uma nova compreensão sobre a natureza da felicidade e do bem-estar. Rio de Janeiro: Objetiva, 2011.

SELIGMAN, M. E. The optimistic child: a proven program to safeguard children against depression and build lifelong resilience. [S.I.]: Mariner Books, 2007.

SUOMI. O Kiva na Escola. This is Finland, 2011. Disponivel em: https://finland.fi/pt/vida-amp-sociedade/o-kiva-na-escola/. Acesso em: 15 mar. 2019.

TURKU, U. O. Kiva. KiVa International, 2018. Disponivel em: http://www.kivaprogram.net/. Acesso em: 8 mar. 2019. 
UNGAR, M. Resilience across culture. British journal of social work, v. 38, n. 2, p. 218-235, 2008.

WONG, M. C. S. et al. The impact of a newly designed resilience-enhancing programme on parentand teacher-perceived resilience environment among Health Promoting Schools in Hong Kong. Journal of Epidemiology and Community Health, v. 63, n. 3, p. 209-214., 2009. 